\title{
Shorter ejaculatory abstinence interval and maternal endometrium exposure to seminal plasma as tools to improve pregnancy rate in patients undergoing intracytoplasmic sperm injection cycles
}

\author{
Edson Borges Jr. ${ }^{1,2}$, Daniela Paes de Almeida Ferreira Braga ${ }^{1,2}$, Amanda Souza Setti ${ }^{1,2}$ \\ ${ }^{1}$ Fertility Medical Group. São Paulo/SP, Brazil \\ ${ }^{2}$ Instituto Sapientiae - Centro de Estudos e Pesquisa em Reprodução Assistida. São Paulo/SP, Brazil
}

Infertility affects nearly $20 \%$ of couples in reproductive age, and male factor of infertility is accountable for roughly half of the cases (Ricci et al., 2017). Despite significant progress has been made in the diagnosis and treatment of male infertility, which allowed many men to have a biological child, the causes of many cases of male infertility are still unknown, thus hampering the use of tools to correct them.

The general semen quality seems to be deteriorating over time (Borges et al., 2015) and there are only so many things that can be done concerning the male factor of infertility. To date, the intracytoplasmic sperm injection (ICSI) is the treatment of choice for couples with male factor infertility, since the technique bypasses the sperm limitations related to the fertilization process and subsequent establishment of pregnancy. Nevertheless, according to recent studies, simple recommendations may favor the odds of pregnancy, such as shorter ejaculatory abstinence (EA) interval (Periyasamy et al., 2017) and maternal endometrium exposure to paternal seminal plasma (Crawford et al., 2015).

An EA interval of 2-7 days and 3-4 days prior to semen collection for conventional semen analysis has been recommended by the World Health Organization guidelines (World Health Organization, 2010) and the European Society of Human Reproduction and Embryology (Kvist \& Björndahl, 2002), respectively. A recent study confirmed that an EA period of more than 7 days adversely impacts the outcomes, and that an abstinence of 2-4 days is associated with higher live-birth rates compared with periods of more than 7 days (Periyasamy et al., 2017). In a study performed at out center, prolonged EA intervals had a detrimental effect on sperm function and quality. Despite Ionger EA intervals resulted in higher semen volume, sperm count, and total motile sperm count, they also increased sperm DNA fragmentation index and decreased fertilization, blastocyst formation rate, implantation rate and pregnancy rate. Additionally, the study established a cutoff point for EA length at 4 days (unpublished results). We postulated that shortening of EA intervals could optimize sperm quality by keeping epidydimal stasis at a minimum.

A recent meta-analysis of 2,204 patients in seven random controlled trials found a $23 \%$ improvement in clinical pregnancy rates when maternal endometria were exposed to seminal plasma around the time of oocyte retrieval or embryo transfer (Crawford et al., 2015). Initially, seminal fluid, which is a cocktail complex cocktail of functionally diverse substances such as antioxidants, buffers, hormones, proteins and sugars, was simply presumed to facilitate sperm survival and delivery to the female reproductive tract. At present, the importance of these seminal fluid components as potential mediators of paternal-maternal communication has begun to be recognized (Hopkins et al., 2017).

Studies over the past two decades in both animal and human models showed that seminal plasma may positively affect endometrial receptivity, inducing an inflammation-like response, and thus promotes immune tolerance, facilitates implantation, and even positively influences offspring health (Bellinge et al., 1986; Tremellen et al., 2000; Kho et al., 2009; Robertson \& Sharkey, 2016). Previous studies showed that pregnancy outcomes are higher (i) after intravaginal administration of seminal plasma at embryo or gamete intrafallopian transfer (GIFT) (Bellinge et al., 1986), (ii) in couples that had sexual intercourse close to the time of human chorionic gonadotropin administration in GIFT cycles (Marconi et al., 1989), (iii) in women experiencing recurrent miscarriage with pessaries of donor seminal plasma (Coulam \& Stern, 1995), and (iv) after in vitro fertilization treatment (IVF) when couples have intercourse either just before or just after embryo transfer (Tremellen et al., 2000). Still, there are some points that need to be addressed in future works, such as whether there is a superior method of seminal fluid exposure (sexual intercourse or delivery of intravaginal, intracervical, or intrauterine administration), and whether the general recommendation to patients undergoing IVF treatment, which are regularly counseled to abstain from coital activity because intercourse may cause uterine contractions and impair embryo implantation, is outdated.

Shortening EA interval and maternal exposure to paternal seminal plasma may be considered potential approaches for improving ICSI outcomes. Data provided here are promising, but requires well-designed studies to evaluate effects on pregnancy in patients undergoing ICSI as a result of male factor of infertility, before routine incorporation into clinical practice.

\section{REFERENCES}

Bellinge BS, Copeland CM, Thomas TD, Mazzucchelli RE, O'Neil G, Cohen MJ. The influence of patient insemination on the implantation rate in an in vitro fertilization and embryo transfer program. Fertil Steril. 1986;46:252-6. PMID: 3732531 DOI: 10.1016/S0015-0282(16)49521-X

Borges E Jr, Setti AS, Braga DP, Figueira R de C, Iaconelli A Jr. Decline in semen quality among infertile men in Brazil during the past 10 years. Int Braz J Urol. 2015;41:75763. PMID: 26401870 DOI: $10.1590 / S 1677-5538$. IBJU.2014.0186

Coulam CB, Stern JJ. Effect of seminal plasma on implantation rates. Early Pregnancy. 1995;1:33-6. PMID: 9363233

Crawford G, Ray A, Gudi A, Shah A, Homburg R. The role of seminal plasma for improved outcomes during in vitro fertilization treatment: review of the literature and meta-analysis. Hum Reprod Update. 2015;21:275-84. PMID: 25281684 DOI: $10.1093 /$ humupd/dmu052 
Hopkins BR, Sepil I, Wigby S. Seminal fluid. Curr Biol. 2017;27:R404-R405. PMID: 28586660 DOI: 10.1016/j. cub.2017.03.063

Kho EM, McCowan LM, North RA, Roberts CT, Chan E, Black MA, Taylor RS, Dekker GA; SCOPE Consortium. Duration of sexual relationship and its effect on preeclampsia and small for gestational age perinatal outcome. J Reprod Immunol. 2009;82:66-73. PMID: 19679359 DOI: 10.1016/j. jri.2009.04.011

Kvist U, Björndahl L, eds. Manual on Basic Semen Analysis. ESHRE Monographs. Oxford: Oxford University Press; 2002.

Marconi G, Auge L, Oses R, Quintana R, Raffo F, Young E. Does sexual intercourse improve pregnancy rates in gamete intrafallopian transfer? Fertil Steril. 1989;51:357-9. PMID: 2643533 DOI: 10.1016/S0015-0282(16)60507-1

Periyasamy AJ, Mahasampath G, Karthikeyan M, Mangalaraj AM, Kunjummen AT, Kamath MS . Does duration of abstinence affect the live-birth rate after assisted reproductive technology? A retrospective analysis of 1,030 cycles. Fertil Steril. 2017;108:988-92. PMID: 29100624 DOI: $10.1016 /$ j.fertnstert.2017.08.034
Ricci E, Viganò P, Cipriani S, Somigliana E, Chiaffarino F, Bulfoni A, Parazzini F. Coffee and caffeine intake and male infertility: a systematic review. Nutr J. 2017;16:37. PMID: 28646871 DOI: 10.1186/s12937-017-0257-2

Robertson SA, Sharkey DJ. Seminal fluid and fertility in women. Fertil Steril. 2016;106:511-9. PMID: 27485480 DOI: $10.1016 /$ j.fertnstert.2016.07.1101

Tremellen KP, Valbuena D, Landeras J, Ballesteros A, Martinez J, Mendoza S, Norman RJ, Robertson SA, Simón C. The effect of intercourse on pregnancy rates during assisted human reproduction. Hum Reprod. 2000;15:2653-8. PMID: 11098040 DOI: 10.1093/humrep/15.12.2653

World Health Organization. WHO laboratory manual for the examination and processing of human semen. 5th ed. Geneva: World Health Organization; 2010. 287 p. 